\title{
Sensing Optimization for an Receiver Structure in Cognitive Radio Systems
}

\author{
Bub-Joo Kang, Yoon-Seok Nam, Member, KIMICS
}

\begin{abstract}
This paper describes the optimization of spectrum sensing in terms of the throughput of a cognitive radio $(C R)$ system. Dealing with the optimization problem of spectrum sensing, this paper evaluates the throughput of a CR system by considering such situations as the penalty time of a channel search and incumbent user (IU) detection delay caused by a missed detection of an incumbent signal. Also, this paper suggests a serial channel search scheme as the search method for a vacant channel, and derives its mean channel search time by considering the penalty time due to the false alarm of a vacant channel search. The numerical results suggest the optimum sensing time of the channel search process using the derived mean channel search time of a serial channel search in the case of a sensing hardware structure with single radio frequency (RF) path. It also demonstrates that the average throughput is improved by two separate RF paths in spite of the hardware complexity of an $\mathrm{RF}$ receiver.
\end{abstract}

Index Terms - Spectrum sensing, sensing optimization, vacant channel search, cognitive radio (CR).

\section{INTRODUCTION}

The licensed spectrum allocation method appears to have low spectrum utilization in many parts of the frequency band. In the Federal Communications Commission (FCC) definition, a cognitive radio (CR) system is a spectrum utilization that allows CR users to use spectrum licensed to incumbent users when they are inactive [1]-[2]. In order to utilize the inactive frequency bands, CR users must perform the spectrum sensing for the CR-used frequency bands frequently or periodically. The main reasons for spectrum sensing in CR users can be classified into two types. The first is to gather vacant channels for CR users, and the second is to check the appearance of incumbent users for the corresponding channel that a CR user is utilizing. This paper evaluates the sensing performance in terms of throughput efficiency by considering channel monitoring, a channel search, and a channel switch. The tradeoff between

\footnotetext{
Manuscript received January 7, 2011; revised February 3, 2011; accepted February 7, 2011.

Bub-Joo Kang and Yoon-Seok Nam are with the Department of Information and Communication Engineering, Dongguk University, Gyeongju, 780-714, Korea (Email: bjkang@dongguk.ac.kr and ysnam@dongguk.ac.kr)
}

sensing time and throughput in a $\mathrm{CR}$ system is an important issue for sensing optimization. Because there is a tradeoff between channel monitoring time and throughput, researches on the optimal sensing time have been performed in terms of throughput or throughput efficiency [3],[4].

This paper evaluates the throughput of a CR user by considering such situations as the penalty time in the channel search process and an incumbent user (IU) detection delay in the channel monitoring process, while conventional researches [3],[4] do not describe the optimization problem of the spectrum sensing considering such situations. This paper also derives the mean channel search time in consideration of the penalty time, which is caused by the false alarm created in the search process of a vacant channel. Also, if the CR system does not detect incumbent users during $\mathrm{CR}$ operation during the existence of an incumbent signal, this paper suggests the IU detection delay as the delay time caused by the continuous channel monitoring due to a misdetection. When the CR system continues to perform a $\mathrm{CR}$ operation during the existence of incumbent users, both the CR and incumbent users are affected by interference from each other. In such an event, this paper assumes that transmission errors in CR systems can occur without a successful data reception. Thus the throughput of the CR system in this case is almost zero.

This paper also considers a sensing structure with two separate RF paths for the data transceiver and sensing receiver [5], and compares its sensing performance with that of a single RF path in terms of CR system throughput.

This paper is organized as follows. In section II, the sensing optimization is analyzed in terms of mean channel search time of the serial channel search and average throughput. Numerical results are presented in section III, and finally a conclusion is offered in section IV.

\section{SENSING OPTIMIZATION ANALYSIS}

In order to use the license band of incumbent users, CR users must investigate the vacancy of frequency channels using spectrum sensing.

Figure 1 shows the procedure of vacant channel utilization and periodic spectrum sensing in a CR system. 
In the figure, the channel availability check duration, $\mathrm{T}_{\text {check, }}$, is the time during which a CR system searches vacant channels for $\mathrm{CR}$ network initialization. When a $\mathrm{CR}$ system completes a search of vacant channels initially, the CR system sets up a CR-operating channel by selecting one of the vacant channels for data communication. The CR users perform the spectrum sensing to identify the appearance of an incumbent user through a channel monitoring process after a channel is setup, as shown in Fig. 1.

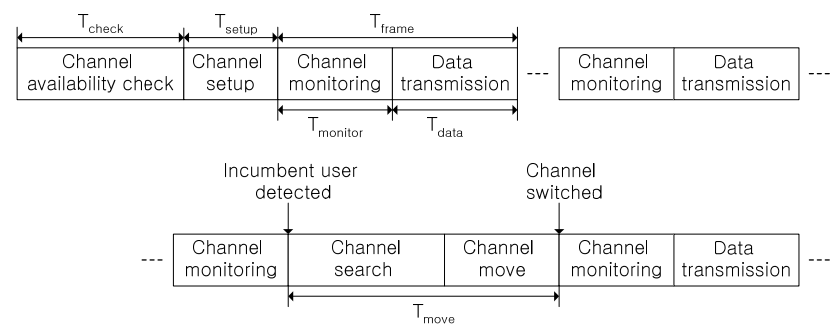

Fig.1. Procedure of vacant channel utilization and periodic spectrum sensing in a CR system.

The channel monitoring process to identify the appearance of incumbent users is performed periodically using sensing parameters such as sensing period, $\mathrm{T}_{\text {frame, }}$ and sensing time, $T_{\text {monitor, }}$, as shown in Fig. 1. Then, if an incumbent signal appears in the frequency channel corresponding to the CR-operating channel, the CR user must switch to another vacant channel to be assigned as a backup channel. Here, the backup channel is defined as a vacant channel that can be assigned as a CR-operating channel during the channel switch process. This paper evaluates the sensing performance by considering the channel search, channel switch, and channel monitoring processes, as shown in Fig. 1.

Also, for the appearance of an incumbent signal, the conventional research analyzes the sensing performance for cases in which a CR system stops its CR operation and searches other vacant channels. In this paper, however, owing to the sensing structure of two separate RF paths as shown in Fig. 2, the channel switch time shortens by searching vacant channels during data transmission time, $\mathrm{T}_{\text {data. }}$.

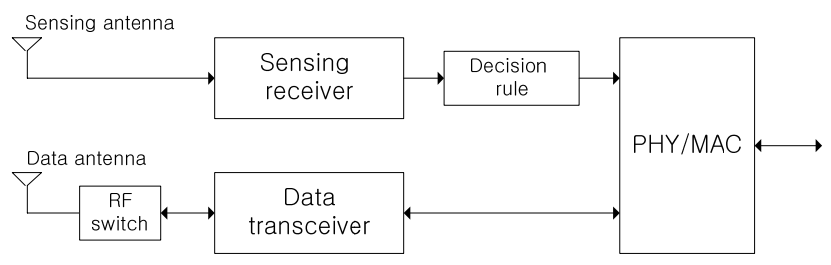

Fig. 2. Sensing structure of two separate RF paths in a CR system.
In Fig. 2, since the CR terminal and CR base station do not know the appearance position of an incumbent user, a spectrum sensing antenna is used as an omni-directional antenna. However, in order to reduce the interference of signals other than those from the CR system, the data antenna for $\mathrm{CR}$ communications is composed of a directional antenna. Thus, in order to obtain the directional antenna gain of the data antenna, the sensing structure with two separate RF paths has an advantage in terms of the sensing antenna and data antenna configuration, as shown in Fig. 2.

For cases when an incumbent signal appears during a CR operation, the previous research proposes a method in which the CR user searches another vacant channel after determining the appearance of the incumbent signal [3]. A state diagram of the serial channel search scheme for a serial channel search performed on $\mathrm{N}$ frequency channels, except for the $k$-th CR-operating channel, when the number of total frequency channels in the CR system is given as $\mathrm{N}+1$, is shown in Fig. 3.

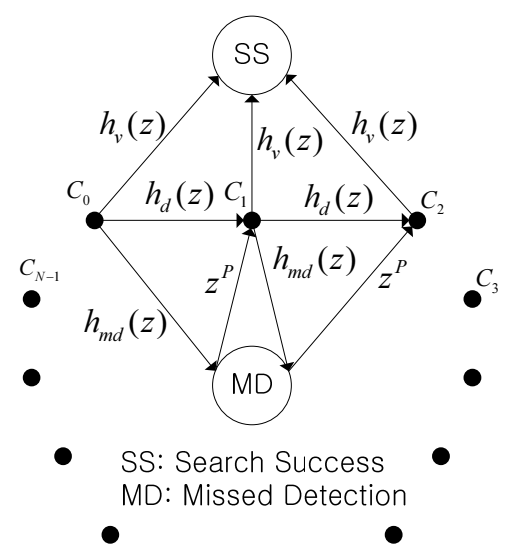

Fig. 3. State diagram of the serial channel search process.

In Fig. 3, a search success (SS) state is defined as the state that acquires a vacant channel successfully, while a missed detection (MD) state is described as a case in which a vacant channel is declared incorrectly due to a false alarm on the existence of an incumbent signal when such an incumbent signal exists. From Fig. 3, $C_{i}, i=0,1, \ldots, N-1$ is the $i$-th frequency channel, $h_{v}(z)$ is the path function that acquires the vacant channel successfully, and $h_{d}(z)$ is the path function that detects correctly the existence of an incumbent signal. Finally $h_{m d}(z)$ is the path function that declares incorrectly a vacant channel during the existence of an incumbent signal, and $z^{P}$ is the penalty time that is given by a false alarm of a vacant channel due to a missed detection of an incumbent signal in the channel search process.

From Fig. 3, $h_{v}(z), h_{d}(z)$, and $h_{m d}(z)$ are represented by 


$$
\begin{array}{r}
h_{v}(z)=P\left(H_{0}\right)\left(1-P_{f}^{s}\right) z, \\
h_{d}(z)=\left\{P\left(H_{0}\right) P_{f}^{s}+P\left(H_{1}\right) P_{d}^{s}\right\} z, \\
\text { and } \\
h_{m d}(z)=P\left(H_{1}\right)\left(1-P_{d}^{s}\right) z,
\end{array}
$$

where $P\left(H_{0}\right)$ is defined as the probability that an incumbent signal is inactive, and $P\left(H_{1}\right)$ is defined as the probability that an incumbent signal is active, $P\left(H_{0}\right)+P\left(H_{1}\right)=1[4] . P_{d}^{s}$ and $P_{f}^{s}$ are the probabilities of a signal detection and false alarm in the channel search process, respectively. From Fig. 3 , if $h_{v}(z)$ is the success function of the channel search of a vacant channel and $h_{n v}(z)$ is the failure function of the channel search of a vacant channel, $h_{v}(z)$ and $h_{n v}(z)$ are given by

$$
\begin{aligned}
h_{n v}(z) & =h_{d}(z)+h_{m d}(z) z^{P} \\
& =\left\{P\left(H_{0}\right) P_{f}^{s}+P\left(H_{1}\right) P_{d}^{s}\right\} z+P\left(H_{1}\right)\left(1-P_{d}^{s}\right) z^{P+1}
\end{aligned}
$$

In Fig. 3, performing a serial channel search for $\mathrm{N}$ available channels, the success function of the channel search, $H_{V}(z)$ is given by

$$
\begin{aligned}
H_{V}(z)= & h_{v}(z)+h_{n v}(z) h_{v}(z)+h_{n v}^{2}(z) h_{v}(z)+\ldots \\
& +h_{n v}^{N-1}(z) h_{v}(z) \\
= & h_{v}(z) \sum_{i=0}^{N-1} h_{n v}^{i}(z)
\end{aligned}
$$

The transfer function that starts a channel search at the $i$-th channel is given by

$$
U_{i}(z)=\frac{H_{V}(z)}{1-h_{n v}^{N}(z)}
$$

From Fig. 3, since all channels are equally likely a priori, the total transfer function averaged over all $\mathrm{N}$ frequency channels is represented by

$$
\begin{aligned}
U(z) & =\frac{1}{N} \sum_{i=0}^{N-1} U_{i}(z) \\
& =\frac{H_{V}(z)}{1-h_{n v}^{N}(z)}
\end{aligned}
$$

Thus, the mean channel search time of the serial channel search scheme is given by

$$
\begin{aligned}
\bar{T}_{\text {search }} & =\left.\frac{d}{d z} \ln U(z)\right|_{z=1} T_{\text {search }} \\
& =\left[\frac{H_{V}^{\prime}(1)}{H_{V}(1)}+\frac{N h_{n v}^{\prime}(1) h_{n v}^{N-1}(1)}{1-h_{n v}^{N}(1)}\right] T_{\text {search }},
\end{aligned}
$$

where $T_{\text {search }}$ is the sensing time per one channel in the channel search process, $H(1)=\left.H(z)\right|_{z=1}, \quad h(1)=\left.h(z)\right|_{z=1}$, $H^{\prime}(1)=\left.\frac{d}{d z} H(z)\right|_{z=1}$, and $h^{\prime}(1)=\left.\frac{d}{d z} h(z)\right|_{z=1}$.

The sensing structure of a single RF path for sensing and CR operation performs both the channel search and channel switch processes during the appearance of incumbent users, while the sensing structure of two separate RF paths conducts only the channel switch process due to the acquirement of vacant channels using the data transmission time. Thus, the channel movement time for the sensing structure with two separate RF paths is given by

$$
\bar{T}_{\text {move }}=T_{\text {frame }}+T_{\text {swich }}
$$

And the channel movement time for the sensing structure with a single RF path is given by

$$
\bar{T}_{\text {move }}=T_{\text {frame }}+\bar{T}_{\text {search }}+T_{\text {switch }}
$$

In general, the throughput is defined as a ratio of the data transmission time divided by the total channel occupation time of the CR-operating channel. From Fig. 2, the total channel occupation time of the CR-operating channel, $\bar{T}_{\text {total }}$ is given by

$$
\begin{aligned}
\bar{T}_{\text {total }}= & P\left(H_{0}\right)\left(1-P_{f}^{m}\right) T_{\text {frame }}+P\left(H_{0}\right) P_{f}^{m} \bar{T}_{\text {move }} \\
& +P\left(H_{1}\right) P_{d}^{m} \bar{T}_{\text {move }}+P\left(H_{1}\right)\left(1-P_{d}^{m}\right) T_{\text {delay }},
\end{aligned}
$$

where $P_{d}^{m}$ and $P_{f}^{m}$ are the probability of signal detection and the probability of a false alarm in the channel monitoring process, respectively. Also, $T_{\text {delay }}$ is the IU detection delay, which is defined as a transmission delay caused by a transmission error due to the interference of an incumbent signal when detection of the incumbent signal is missed. $T_{\text {delay }}$ can be described as a transmission delay due to a missed detection for continuous channel monitoring when a transmission error occurs at the CR-operating channel. The detection of the incumbent signal can also be determined by a rapid increase of bit error rate measured by $\mathrm{M}$ data frames when a missed detection of an incumbent signal occurs during 
the continuous channel monitoring process. In such a case, $\mathrm{T}_{\text {delay }}$ is represented as $\mathrm{MT}_{\text {frame }}$.

In this paper, the derivation of throughput considering the serial channel search process and IU detection delay is as follows. The mean data transmission time of the CRoperating channel is given by

$$
\bar{T}_{\text {data }}=T_{\text {data }}\left\{P\left(H_{0}\right)\left(1-P_{f}^{m}\right)+P\left(H_{1}\right)\left(1-P_{d}^{m}\right)\right\}
$$

In (12), the throughput for the appearance of an incumbent signal is almost zero due to the transmission error caused by the interference of the incumbent signal. Thus, the mean data transmission time can be represented by

$$
\bar{T}_{\text {data }} \simeq T_{\text {data }} P\left(H_{0}\right)\left(1-P_{f}^{m}\right)
$$

Finally, the average throughput using (11) and (13) can be represented by

$$
\begin{aligned}
\bar{\eta} & =\frac{\bar{T}_{\text {data }}}{\bar{T}_{\text {toual }}} \eta \\
\simeq & \frac{\bar{T}_{\text {data }} P\left(H_{0}\right)\left(1-P_{f}^{m}\right)}{P\left(H_{0}\right)\left\{\left(1-P_{f}^{m}\right) T_{\text {frame }}+P_{f}^{m} \bar{T}_{\text {move }}\right\}+P\left(H_{1}\right)\left\{P_{d}^{m} \bar{T}_{\text {move }}+\left(1-P_{d}^{m}\right) T_{\text {delay }}\right\}} \eta
\end{aligned},
$$

where $\eta$ is the data rate of the CR system.

\section{NUMERICAL RESULTS}

In this paper, an incumbent signal for the numerical results is assumed to be a binary phase-shift keying signal with a $10 \mathrm{kHz}$ bandwidth. Energy detection is selected for the incumbent signal, and the sampling rate of the energy detector is assumed to be $10 \mathrm{kHz}$. The channel model of both the incumbent and CR systems is assumed to be a Rayleigh fading channel. Considering the Rayleigh fading channel [6],[7], the probabilities of signal detection and a false alarm during the channel monitoring and channel search are given by

$$
\begin{aligned}
& P_{d}^{i}=e^{-\lambda / V_{F}} \sum_{k=0}^{L-1} \frac{\left(\lambda / V_{F}\right)^{k}}{k !}, i=s, m, \\
& P_{f}^{i}=e^{-\lambda / V_{N}} \sum_{k=0}^{L-1} \frac{\left(\lambda / V_{N}\right)^{k}}{k !}, i=s, m,
\end{aligned}
$$

where $\mathrm{L}$ is the time-bandwidth product, $\mathrm{BT}$ the channel bandwidth, $\mathrm{B}$ is multiplied by the sensing time $\mathrm{T}$, and $\lambda$ and $V_{N}$ are the detection threshold and noise variance, respectively. $V_{F}$ is given by

$$
V_{F}=V_{N}(1+\bar{\mu}) \text {, }
$$

where $\bar{\mu}$ is the average SNR of the incumbent signal for the Rayleigh fading channel.

The number of the frequency channels is assumed to be 31 with a bandwidth of $10 \mathrm{kHz}$ in the operating bandwidth of the CR system. The frame length of the CR system, $\mathrm{T}_{\text {frame }}$, is assumed to be $100 \mathrm{~ms}$, and the channel switch time, $T_{\text {switch }}$, is then assumed to $10 \mathrm{~ms}$ for the control signal transmission and channel switch.

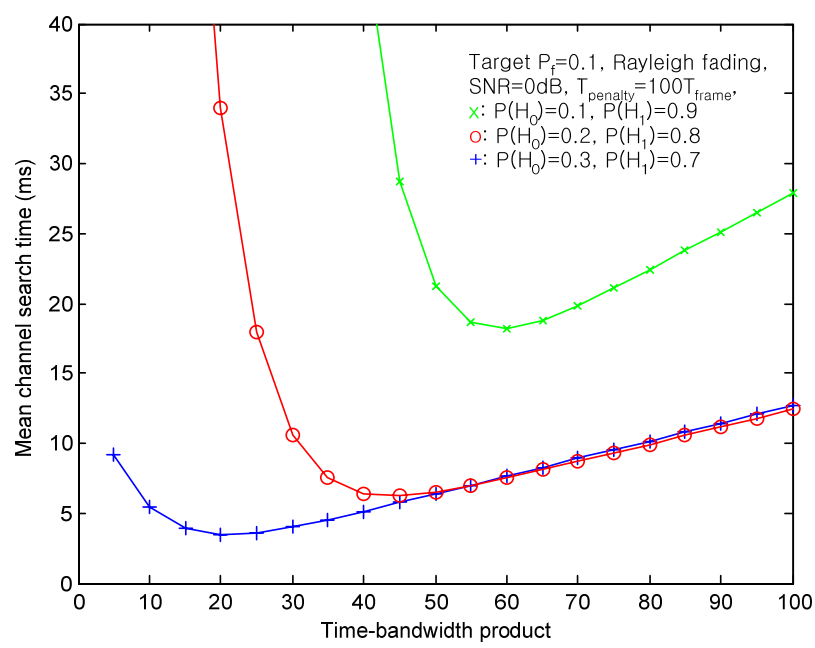

Fig. 4. Mean channel search time for the time-bandwidth product.

Figure 4 shows the mean channel search time for the sensing structure with single RF path when the penalty time is given as $100 \mathrm{~T}_{\text {frame. }}$. In this figure, the changes of mean channel search time for the time-bandwidth product are largely shown when $P\left(H_{0}\right)$ is small and $P\left(H_{1}\right)$ is relatively large. Thus, the selection of the optimal channel search time is an important issue when $P\left(H_{0}\right)$ is less than 0.2 as shown in Fig. 4.

Figure 5 shows the average throughput for the channel monitoring time according to the sensing hardware structure and changes of channel search time. In Fig. 5, the solid lines represent the results for the sensing hardware related to spectrum sensing and data communication in the case of two separate RF paths, while the dotted lines are those for a single RF path. Actually, the average throughput for the channel monitoring time according to the sensing hardware structure has a large difference when the sensing time of the channel monitoring and channel search enlarges. 


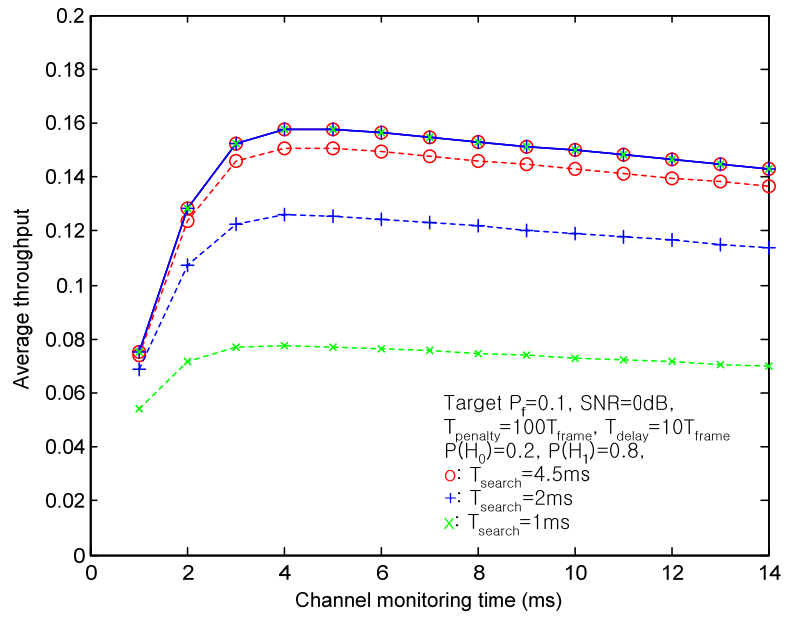

Fig. 5. Channel monitoring time vs. average throughput according to changes of channel search time.

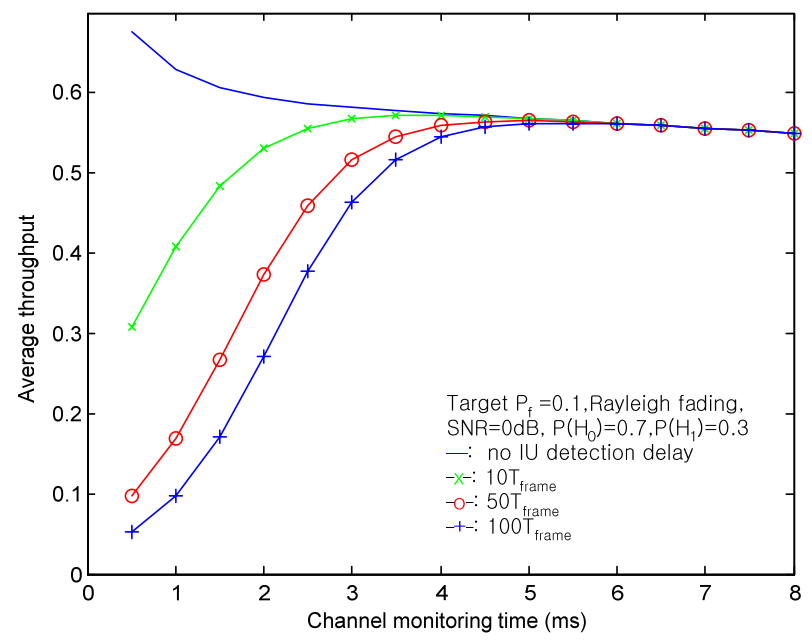

Fig. 6. Channel monitoring time vs. average throughput according to IU detection delay.

Figure 6 shows the average throughput for the channel monitoring time according to the IU detection delay. In Fig. 6, an IU detection delay is defined as a transmission delay caused by a transmission error due to interference of an incumbent signal when detection of the incumbent signal is missed. From (14), if the average throughput is evaluated with no IU detection delay, as shown in Fig. 6, an analytical error is caused as a result of the throughput improvement in cases of shorter channel monitoring times. In Fig. 6, the changes of average throughput for the IU detection delay are very small when the channel monitoring time is assigned as $5 \mathrm{~ms}$ or more.

\section{CONCLUSION}

This paper handles the optimization of spectrum sensing in terms of CR system throughput. This paper also evaluates the throughput of a CR system by considering such situations as channel search, channel switch, and sensing structure, and derives the mean channel search time of a serial channel search by considering the penalty time due to a search error. The numerical results suggest an optimum sensing time of the channel search process using the derived mean channel search time of a serial channel search. It also demonstrates that the average throughput is improved by two separate RF paths in spite of the hardware complexity of the $\mathrm{RF}$ receiver.

\section{ACKNOWLEDGMENT}

This research was financially supported by the Ministry of Education, Science Technology (MEST) and Korea Institute for Advancement of Technology (KIAT) through the Human Resource Training Project for Regional Innovation.

\section{REFERENCES}

[1] IEEE 802.22, Functional Requirements for the 802.22 WRAN Standard, IEEE 802.22-05/007r46, September 2005.

[2] IEEE 802.22 WG, Draft standard for wireless regional area networks part 22, IEEE P802.22/ D0.3, May 2007.

[3] A. Ghasemi and E.S. Sousa, "Optimization of spectrum sensing for opportunistic spectrum access in cognitive radio networks," IEEE CCNC, pp.1022-1026, Jan. 2007.

[4] Y.C. Liang, Y. Zeng, E. Peh, et al., "Sensing-throughput tradeoff for cognitive radio networks, “ IEEE Trans. Wireless Commun. vol. 7, no. 4, pp.1326 1337, April 2008.

[5] G. Chouinard, CPE RF Architecture, IEEE 802.22-07/0081r0, January 2007.

[6] A.J. Viterbi, CDMA Principles of Spread Spectrum Multiple Access Communication, Addison-Wesley, New York, 1995.

[7] H. Urkowitz, "Energy detection of unknown deterministic signals," Proc. IEEE, vol.55, pp.523-531, April 1967.

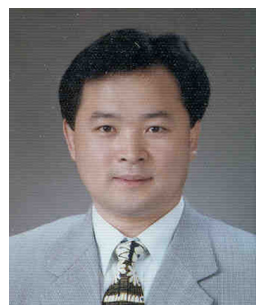

Bub-Joo Kang received the B.S. degree in electronic engineering from Kyunghee University, Seoul, Korea in 1983, and the M.S. and Ph.D. degrees in electronic engineering from Yonsei University, Seoul, Korea in 1985 and 1996, respectively. From 1988 to 2001, he worked for ETRI and served as a principal member of engineering staff at the Modem Technology Department, managing the project of design and development of radio modem technology for IMT2000. He is currently an associate professor in department of information and communications engineering, Dongguk University, Kyungbuk, Korea. His current research interests include cognitive radio, spectrum sensing, OFDM, CDMA, etc.

Yoon-Seok Nam received the B.S., M.S. and Ph.D. degrees in electronic engineering from Kyungbuk National University, Kyungbuk, Korea in 1985, 1987, and 1995, respectively. From 1987 to 2000, he worked for ETRI and served as a principal member of engineering staff. He is currently a professor in department of information and communications engineering, Dongguk University, Kyungbuk, Korea. 